\title{
Risk of Myocardial Infarction and Stroke after Acute Infection or Vaccination
}

\author{
Liam Smeeth, Ph.D., Sara L. Thomas, Ph.D., Andrew J. Hall, Ph.D., \\ Richard Hubbard, D.M., Paddy Farrington, Ph.D., and Patrick Vallance, M.D.
}

\section{ABSTRACT}

\section{BACK GROUND}

There is evidence that chronic inflammation may promote atherosclerotic disease. We tested the hypothesis that acute infection and vaccination increase the short-term risk of vascular events.

\section{METHODS}

We undertook within-person comparisons, using the case-series method, to study the risks of myocardial infarction and stroke after common vaccinations and naturally occurring infections. The study was based on the United Kingdom General Practice Research Database, which contains computerized medical records of more than 5 million patients.

\section{RESULTS}

A total of 20,486 persons with a first myocardial infarction and 19,063 persons with a first stroke who received influenza vaccine were included in the analysis. There was no increase in the risk of myocardial infarction or stroke in the period after influenza, tetanus, or pneumococcal vaccination. However, the risks of both events were substantially higher after a diagnosis of systemic respiratory tract infection and were highest during the first three days (incidence ratio for myocardial infarction, 4.95; 95 percent confidence interval, 4.43 to 5.53; incidence ratio for stroke, 3.19; 95 percent confidence interval, 2.81 to 3.62). The risks then gradually fell during the following weeks. The risks were raised significantly but to a lesser degree after a diagnosis of urinary tract infection. The findings for recurrent myocardial infarctions and stroke were similar to those for first events.

\section{CONCLUSIONS}

Our findings provide support for the concept that acute infections are associated with a transient increase in the risk of vascular events. By contrast, influenza, tetanus, and pneumococcal vaccinations do not produce a detectable increase in the risk of vascular events.
From the Departments of Epidemiology and Population Health (L.S.) and Infectious and Tropical Diseases (S.L.T., A.J.H.), London School of Hygiene and Tropical Medicine, London; the Division of Respiratory Medicine, University of Nottingham, Nottingham (R.H.); the Division of Statistics, Open University, Milton Keynes (P.F.); and the Centre for Clinical Pharmacology, British Heart Foundation Laboratories, Division of Medicine, University College London (P.V.) - all in the United Kingdom. Address reprint requests to $\mathrm{Dr}$. Smeeth at the Department of Epidemiology and Population Health, London School of Hygiene and Tropical Medicine, Keppel St., London WCIE 7HT, United Kingdom, or at liam. smeeth@lshtm.ac.uk.

N Engl J Med 2004;351:2611-8.

Copyright $(2004$ Massachusetts Medical Society. 
YSTEMIC INFLAMMATION AND INFECtions accelerate atherogenesis in animals, and circulating markers of inflammation, such as C-reactive protein, predict the risk of vascular events in humans. ${ }^{2,3}$ However, systemic inflammation is not a constant but varies in response to infections or to other proinflammatory stimuli. Such intermittent changes may be linked to an increase in the risk of vascular events. Indeed, inflammatory markers predict the outcome in acute vascular events ${ }^{4,5}$; an increased leukocyte count may herald a short period of increased risk of stroke $e^{6}$; and several small studies have suggested that there may be a transient increase in the risk of a myocardial infarction after infection..$^{7-12}$

The mechanisms by which acute inflammation may affect the risk of vascular events are uncertain but may include endothelial dysfunction. Such dysfunction is a feature of the increased risk induced by conventional risk factors, ${ }^{13,14}$ and in an experimental model, the vaccination of healthy volunteers induced a short-lived inflammation that was associated with profound suppression of endothelium-dependent relaxation. ${ }^{15}$ If the likelihood of a vascular event is related to variations in the underlying inflammatory state and endothelial function, many naturally occurring common infections or even vaccination could be associated with a shortlived increase in the event rate. To test this hypothesis, we studied the incidence of myocardial infarction and stroke after influenza and other vaccinations or after naturally occurring infections, using the United Kingdom General Practice Research Database (GPRD) and the self-controlled case-series method. ${ }^{16}$

METHO D S
DATABASE
The GPRD has been described in detail elsewhere. ${ }^{17}$
It is the largest source of continuous data on ill-
ness and prescribing habits in the United King-
dom. ${ }^{17}$ The database is representative of all prac-
tices in England and Wales in terms of geographic
distribution and size, and the age and sex distri-
butions of the population included in it are simi-
lar to those of the whole population of the United
Kingdom.
tabase ${ }^{18}$ The information obtained for the da-
study from the scientific and ethics advisory group
of the GPRD.

\section{PARTICIPANTS}

The source population was all patients who were registered for at least one year with a general practice that contributed to the GPRD between 1987 and 2001 (i.e., a total of 5,767,499 people from 687 general practices). The patients had received one or two new diagnoses of myocardial infarction or stroke during the period of at least six months after the start of their follow-up in the GPRD. The events documented during the first six months were excluded because of the possibility that they had occurred before the patient joined the GPRD and had been recorded retrospectively. We did not differentiate between ischemic and hemorrhagic stroke, because such differentiation has been shown to be unreliable with the use of the GPRD. ${ }^{19}$ Patients were excluded if they were younger than 18 years of age at the time of a first myocardial infarction or stroke recorded in the GPRD or if the data in their medical records indicated that the vascular event was likely to have been recorded retrospectively. Examples of retrospectively recorded data include the date of a myocardial infarction or stroke that was the same as the recorded date of other medical events on the date of a new-patient or well-person visit, discharge from the hospital, or a postmortem report or cremation certificate.

\section{EXPOSURE}

Data were extracted on vaccinations against influenza, tetanus, and pneumococcus. Some patients had multiple records within a few days of one another for the same vaccination, indicating that the recorded date of the vaccination was inaccurate. Therefore, for our study, vaccinated persons were restricted to those for whom an influenza vaccination was recorded on a single day within the vaccination season (September 1 to March 31), tetanus vaccinations were received at least three months apart, or a single pneumococcal vaccination was recorded. Data were also extracted on acute urinary tract infections and acute systemic respiratory tract infections such as pneumonia, acute bronchitis, "chest infections," and influenza.

\section{CASE-SERIES METHOD}

Because vaccinated and unvaccinated persons and those with diagnosed infection and those without it differ in ways that are difficult to measure and control for, we undertook within-person comparisons using the case-series method ${ }^{16,20}$ in a population of 
persons who had the outcome of interest. We derived measures of the relative incidence of events within defined intervals after an exposure as compared with all other observed time periods for each person. ${ }^{21}$ The null hypothesis was that vascularevent rates remain constant from day to day and are not affected by an acute exposure to vaccination or infection. The period of exposure was defined as extending up to 91 days after the inflammatory exposure and was subdivided into periods of 1 through 3 days, 4 through 7 days, 8 through 14 days, 15 through 28 days, and 29 through 91 days after the exposure. All other observation time was taken as the baseline period (i.e., without exposure).

Vascular events recorded on the same day as a vaccination or an infection were excluded from the baseline period, because these events may have been recorded retrospectively, when the patient attended the general practice for another reason, as shown in a previous study. ${ }^{22}$ For participants who were exposed to a vaccine or an infection more than once during the observation period, each exposure was followed by a 91-day period in which the participants were at risk. This method and the time intervals used are illustrated in Figure 1. Incidence ratios were calculated for events occurring within each stratum of the period of exposure as compared with the baseline periods.

\section{OUTCOME MEASURES}

We performed separate analyses for the type of event (myocardial infarction or stroke), whether the event was the first of its type or a subsequent event, and the type of inflammatory exposure. A recurrent event was defined as either the first occurrence of the event during the observation period for a participant who had had the same type of event before the start of the observation period or the second of two events that both occurred within the observation period for a participant with no history of such an event. The baseline risk of myocardial infarction or stroke is higher among persons who have had a vascular event. Therefore, the observation period for a recurrent event was restricted to the time after the first event had occurred - either beginning six months after the start of observation of the participant in the GPRD (for the first occurrence of an event during the observation period for a participant who had had the same type of event before the start of the observation period) or beginning with the date of the first event within the observation period (for the second of two events that occurred within the observation period).

The exception to these starting dates was influenza vaccination. One of the indications for administration of this vaccine is preexisting cardiovascular disease, so that the probability of receiving influenza vaccination is itself associated with the risk of vascular events. To ensure that during the observation period there was minimal variation in the opportunity to be vaccinated, the observation period used in the analysis with regard to influenza vaccination did not include the time before a participant's first influenza vaccination.

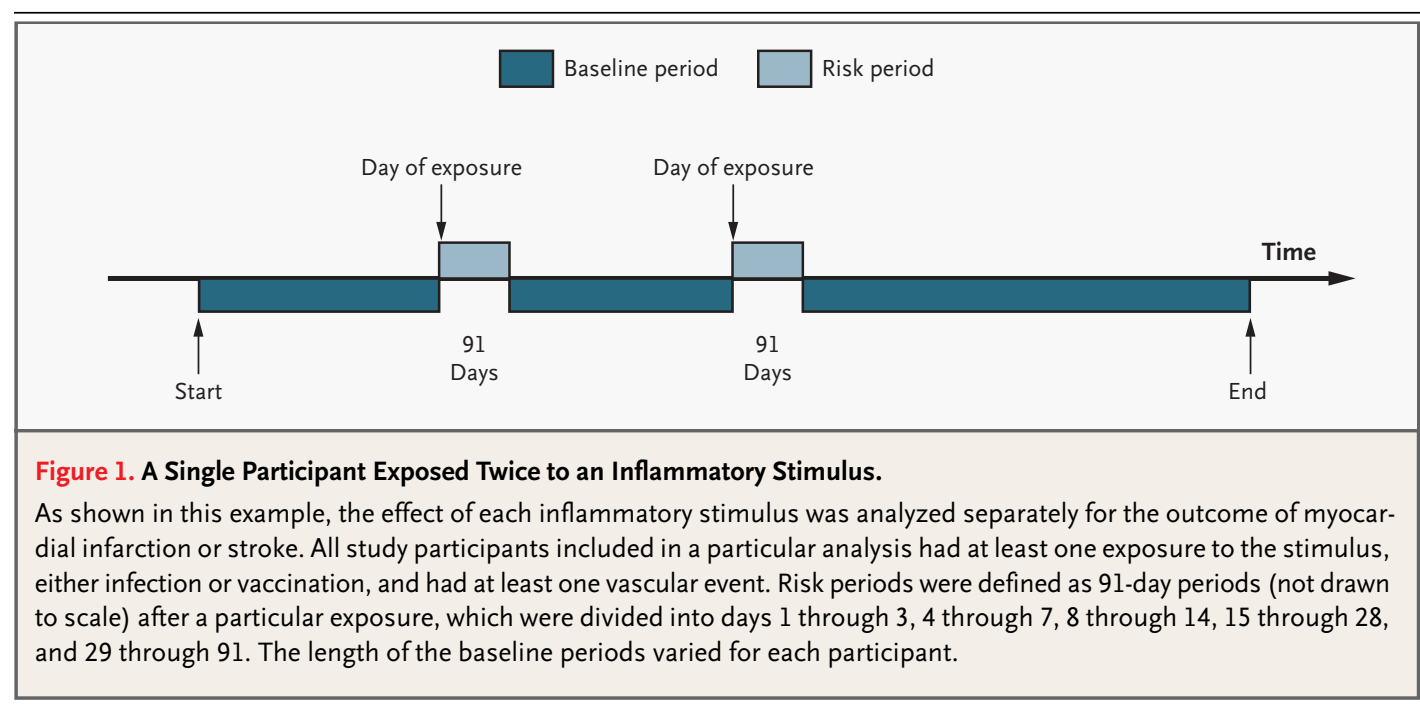


AGE, TIME, AND SECONDARY ANALYSES

Age was controlled for in five-year age groups, and the analysis was repeated with the use of two-year age groups to assess whether finer stratification affected the results. In a case-series analysis, participants who are not exposed to a stimulus at any time during the observation period do not contribute to the estimate of the association between exposure and outcome, but the inclusion of such participants can improve control for confounding by age. Our primary analysis was restricted to participants who were exposed to inflammatory stimuli at least once during the follow-up period, but the analysis was repeated to include all cases in order to ensure that the estimates did not vary. Because the chance of receiving a vaccination during the period immediately after a vascular event may have been different from that during the remainder of the observation period, we repeated the analysis by excluding from the baseline period the three months before each vaccination.

Deaths from cardiovascular disease are more common in winter than in summer. ${ }^{23}$ Respiratory infections occur more commonly in the winter months, and influenza vaccine is usually administered in the early winter. Therefore, a temporal association between vascular events and respiratory infections or influenza vaccination could be observed even in the absence of a causal role. To investigate this possibility, we analyzed separately the effect of respiratory tract infections acquired in warm months (April through September) and in cool months (October through March).

We estimated that the analysis of the risk of myocardial infarction or stroke after influenza vaccination could include around 20,000 participants who had been exposed, a sample size sufficient to provide more than 90 percent power at the 5 percent level of significance to detect a rate ratio of 1.3 during the first three days after exposure.

\section{RESULTS}

A total of 61,556 adults with a first or subsequent myocardial infarction were identified in the GPRD, of whom 1495 were excluded because the date of the myocardial infarction was uncertain. Of the remaining 60,061 patients, 53,709 had had a first myocardial infarction (median age at myocardial infarction, 72.3 years; interquartile range, 62.9 to 80.5 ; male sex, 59.1 percent; mean duration of observation, 5.6 years), and 12,134 had had a recurrent myocardial infarction (median age, 71.8 years; interquartile range, 63.3 to 79.1 ; male sex, 68.8 percent; mean duration of observation, 4.1 years). There were 5782 persons with no history of myocardial infarction before the observation period who had two myocardial infarctions within the observation period and were included in both groups of participants. Of a total of 56,018 adults with a first or subsequent stroke who were identified in the GPRD, 861 were excluded because of an unlikely date of stroke. Of the remaining 55,157 patients, 50,766 had had a first stroke (median age at stroke, 78.3 years; interquartile range, 70.2 to 85.0 ; male sex, 43.8 percent; mean duration of observation, 5.3 years), and 12,804 had had a subsequent stroke (median age, 78.8 years, interquartile range, 71.2 to 85.0 ; male sex, 46.6 percent; mean duration of observation, 3.5 years). Subsequently, 13,099 patients with myocardial infarction and 12,572 with stroke were excluded from one or more of the vaccine analyses because of uncertain vaccination dates.

Only persons who were exposed to either vaccine or infection were included in the primary analysis (Tables 1 and 2). The proportion of participants with a first myocardial infarction or stroke who were exposed to the stimuli of interest ranged from 9 percent (those with stroke who were exposed to pneumococcal vaccine) to 44 percent (those with stroke who were exposed to a systemic respiratory tract infection). The numbers of patients exposed who had a first myocardial infarction or a first stroke and the age-adjusted incidence ratios of a first myocardial infarction or stroke after vaccination and acute infection are shown in Table 1.

There was no increase in the rate of a first myocardial infarction or stroke in the periods after vaccination. However, the rates of both myocardial infarction and stroke were substantially higher after a diagnosis of an acute respiratory tract infection. The rates were highest during the first three days after exposure (i.e., an increase by a factor of nearly five for myocardial infarction and a factor of slightly more than three for stroke) and then fell during the following weeks. The rates also increased after a diagnosis of urinary tract infection.

For second myocardial infarctions or second strokes, again, there was no increase in the rate of events after vaccination (Table 2). The rate of second events after a diagnosis of respiratory tract infection or urinary tract infection followed patterns similar to those observed for first events, although the effect estimates were slightly lower. 


\begin{tabular}{|c|c|c|c|c|c|c|c|c|c|c|}
\hline \multirow[t]{2}{*}{$\begin{array}{l}\text { Outcome and } \\
\text { Risk Period }\end{array}$} & \multicolumn{2}{|c|}{$\begin{array}{l}\text { Influenza Vaccination } \\
\qquad(N=20,486)\end{array}$} & \multicolumn{2}{|c|}{$\begin{array}{l}\text { Tetanus Vaccination } \\
\qquad(\mathrm{N}=7966)\end{array}$} & \multicolumn{2}{|c|}{$\begin{array}{l}\text { Pneumococcal } \\
\text { Vaccination } \\
(\mathrm{N}=5925)\end{array}$} & \multicolumn{2}{|c|}{$\begin{array}{l}\text { Systemic Respiratory } \\
\text { Tract Infection } \\
(\mathrm{N}=\mathbf{2 0 , 9 2 1 )}\end{array}$} & \multicolumn{2}{|c|}{$\begin{array}{l}\text { Urinary Tract Infection } \\
\qquad(\mathrm{N}=10,448)\end{array}$} \\
\hline & $\begin{array}{l}\text { No. of } \\
\text { Cases }\end{array}$ & $\begin{array}{c}\mathrm{IR} \\
(95 \% \mathrm{Cl})\end{array}$ & $\begin{array}{l}\text { No. of } \\
\text { Cases }\end{array}$ & $\begin{array}{c}\mathrm{IR} \\
(95 \% \mathrm{Cl})\end{array}$ & $\begin{array}{l}\text { No. of } \\
\text { Cases }\end{array}$ & $\begin{array}{c}\text { IR } \\
(95 \% \mathrm{Cl})\end{array}$ & $\begin{array}{l}\text { No. of } \\
\text { Cases }\end{array}$ & $\begin{array}{c}\text { IR } \\
(95 \% \mathrm{Cl})\end{array}$ & $\begin{array}{l}\text { No. of } \\
\text { Cases }\end{array}$ & $\begin{array}{c}\text { IR } \\
(95 \% \mathrm{Cl})\end{array}$ \\
\hline \multicolumn{11}{|c|}{ Myocardial infarction } \\
\hline $1-3$ days & 77 & $\begin{array}{c}0.75 \\
(0.60-0.94)\end{array}$ & 12 & $\begin{array}{c}1.10 \\
(0.62-1.92)\end{array}$ & 4 & $\begin{array}{c}0.49 \\
(0.19-1.32)\end{array}$ & 322 & $\begin{array}{c}4.95 \\
(4.43-5.53)\end{array}$ & 58 & $\begin{array}{c}1.66 \\
(1.28-2.14)\end{array}$ \\
\hline 4-7 days & 94 & $\begin{array}{c}0.68 \\
(0.56-0.84)\end{array}$ & 17 & $\begin{array}{c}1.16 \\
(0.72-1.87)\end{array}$ & 12 & $\begin{array}{c}1.11 \\
(0.63-1.96)\end{array}$ & 276 & $\begin{array}{c}3.20 \\
(2.84-3.60)\end{array}$ & 75 & $\begin{array}{c}1.61 \\
(1.28-2.02)\end{array}$ \\
\hline 8-14 days & 176 & $\begin{array}{c}0.73 \\
(0.63-0.85)\end{array}$ & 25 & $\begin{array}{c}0.97 \\
(0.66-1.44)\end{array}$ & 23 & $\begin{array}{c}1.22 \\
(0.81-1.84)\end{array}$ & 422 & $\begin{array}{c}2.81 \\
(2.54-3.09)\end{array}$ & 100 & $\begin{array}{c}1.22 \\
(1.00-1.49)\end{array}$ \\
\hline $15-28$ days & 417 & $\begin{array}{c}0.87 \\
(0.79-0.96)\end{array}$ & 46 & $\begin{array}{c}0.89 \\
(0.66-1.19)\end{array}$ & 43 & $\begin{array}{c}1.15 \\
(0.85-1.55)\end{array}$ & 576 & $\begin{array}{c}1.95 \\
(1.79-2.12)\end{array}$ & 217 & $\begin{array}{c}1.32 \\
(1.16-1.52)\end{array}$ \\
\hline 29-91 days & 2,154 & $\begin{array}{c}1.03 \\
(0.98-1.08)\end{array}$ & 253 & $\begin{array}{c}1.07 \\
(0.94-1.21)\end{array}$ & 177 & $\begin{array}{c}1.10 \\
(0.95-1.28)\end{array}$ & 1,658 & $\begin{array}{c}1.40 \\
(1.33-1.48)\end{array}$ & 820 & $\begin{array}{c}1.23 \\
(1.14-1.33)\end{array}$ \\
\hline \multirow{3}{*}{$\begin{array}{l}\text { Baseline } \\
\text { period }\end{array}$} & 17,533 & 1.00 & 7605 & 1.00 & 5662 & 1.00 & 17,099 & 1.00 & 9079 & 1.00 \\
\hline & \multicolumn{2}{|c|}{$\begin{array}{l}\text { Influenza Vaccination } \\
\qquad(N=19,063)\end{array}$} & \multicolumn{2}{|c|}{$\begin{array}{l}\text { Tetanus Vaccination } \\
(\mathrm{N}=6155)\end{array}$} & \multicolumn{2}{|c|}{$\begin{array}{c}\text { Pneumococcal } \\
\text { Vaccination } \\
(\mathrm{N}=4416)\end{array}$} & \multicolumn{2}{|c|}{$\begin{array}{l}\text { Systemic Respiratory } \\
\text { Tract Infection } \\
(\mathrm{N}=\mathbf{2 2 , 4 0 0 )}\end{array}$} & \multicolumn{2}{|c|}{$\begin{array}{l}\text { Urinary Tract Infection } \\
\qquad(\mathrm{N}=14,603)\end{array}$} \\
\hline & $\begin{array}{l}\text { No. of } \\
\text { Cases }\end{array}$ & $\begin{array}{c}\mathrm{IR} \\
(95 \% \mathrm{Cl})\end{array}$ & $\begin{array}{l}\text { No. of } \\
\text { Cases }\end{array}$ & $\begin{array}{c}\text { IR } \\
(95 \% \mathrm{Cl})\end{array}$ & $\begin{array}{l}\text { No. of } \\
\text { Cases }\end{array}$ & $\begin{array}{c}\text { IR } \\
(95 \% \mathrm{Cl})\end{array}$ & $\begin{array}{l}\text { No. of } \\
\text { Cases }\end{array}$ & $\begin{array}{c}\text { IR } \\
(95 \% \mathrm{Cl})\end{array}$ & $\begin{array}{l}\text { No. of } \\
\text { Cases }\end{array}$ & $\begin{array}{c}\text { IR } \\
(95 \% \mathrm{Cl})\end{array}$ \\
\hline \multicolumn{11}{|l|}{ Stroke } \\
\hline $1-3$ days & 76 & $\begin{array}{c}0.77 \\
(0.61-0.96)\end{array}$ & 11 & $\begin{array}{c}1.33 \\
(0.74-2.41)\end{array}$ & 9 & $\begin{array}{c}1.29 \\
(0.67-2.49)\end{array}$ & 244 & $\begin{array}{c}3.19 \\
(2.81-3.62)\end{array}$ & 152 & $\begin{array}{c}2.72 \\
(2.32-3.20)\end{array}$ \\
\hline 4-7 days & 95 & $\begin{array}{c}0.72 \\
(0.59-0.88)\end{array}$ & 15 & $\begin{array}{c}1.36 \\
(0.82-2.26)\end{array}$ & 10 & $\begin{array}{c}1.08 \\
(0.58-2.01)\end{array}$ & 237 & $\begin{array}{c}2.34 \\
(2.05-2.66)\end{array}$ & 158 & $\begin{array}{c}2.12 \\
(1.81-2.48)\end{array}$ \\
\hline 8-14 days & 194 & $\begin{array}{c}0.84 \\
(0.73-0.96)\end{array}$ & 15 & $\begin{array}{c}0.77 \\
(0.46-1.28)\end{array}$ & 19 & $\begin{array}{c}1.18 \\
(0.75-1.85)\end{array}$ & 368 & $\begin{array}{c}2.09 \\
(1.89-2.32)\end{array}$ & 245 & $\begin{array}{c}1.89 \\
(1.65-2.13)\end{array}$ \\
\hline $15-28$ days & 409 & $\begin{array}{c}0.88 \\
(0.80-0.97)\end{array}$ & 40 & $\begin{array}{c}1.02 \\
(0.74-1.39)\end{array}$ & 29 & $\begin{array}{c}0.90 \\
(0.63-1.30)\end{array}$ & 561 & $\begin{array}{c}1.68 \\
(1.54-1.82)\end{array}$ & 445 & $\begin{array}{c}1.71 \\
(1.55-1.88)\end{array}$ \\
\hline 29-91 days & 2,051 & $\begin{array}{c}1.01 \\
(0.96-1.06)\end{array}$ & 209 & $\begin{array}{c}1.15 \\
(1.00-1.32)\end{array}$ & 160 & $\begin{array}{c}1.15 \\
(0.98-1.35)\end{array}$ & 1,650 & $\begin{array}{c}1.33 \\
(1.26-1.40)\end{array}$ & 1,250 & $\begin{array}{c}1.22 \\
(1.15-1.30)\end{array}$ \\
\hline $\begin{array}{l}\text { Baseline } \\
\text { period }\end{array}$ & 16,188 & 1.00 & 5853 & 1.00 & 4184 & 1.00 & 18,056 & 1.00 & 12,164 & 1.00 \\
\hline $\begin{array}{l}\text { The numbers of } \\
\text { small number } \\
\text { events may have } \\
\text { incidence ratio, }\end{array}$ & $\begin{array}{l}\text { articipan } \\
\text { had a r } \\
\text { een reco } \\
\text { d Cl con }\end{array}$ & $\begin{array}{l}\text { exposed to } \\
\text { orded myoca } \\
\text { ed retrospec } \\
\text { dence interva }\end{array}$ & artici & $\begin{array}{l}\text { accination } \\
\text { on or strok } \\
\text { ce during } \\
\text { s may app }\end{array}$ & $\begin{array}{l}\text { fectio } \\
\text { the da } \\
\text { aselin } \\
\text { ithin }\end{array}$ & $\begin{array}{l}\text { shown in } \\
\text { exposure } \\
\text { iod servec } \\
\text { than one }\end{array}$ & $\begin{array}{l}\text { enthes } \\
\text { was no } \\
\text { he refe } \\
\text { gory. }\end{array}$ & $\begin{array}{l}\text { for each expo } \\
\text { icluded in the } \\
\text { ce category. I }\end{array}$ & $\begin{array}{l}\text { ure. The } \\
\text { analysis } \\
\text { denote }\end{array}$ & $\begin{array}{l}\text { include a } \\
\text { pecause the } \\
\text { age-adjusted }\end{array}$ \\
\hline
\end{tabular}

\section{SECONDARY ANALYSES}

Controlling for age in two-year age groups, including participants who had not been exposed, and excluding the three months before each vaccination made no material difference to the effect estimates (data not shown). There were slightly more participants with a first myocardial infarction $(21,957)$ or stroke $(20,023)$ in winter than in summer $(20,154$ participants with a first myocardial infarction, and
19,148 with a first stroke). However, the significant graded effect of respiratory tract infection remained when the analysis was restricted to events occurring in the summer.

\section{I SCUSSION}

This study shows that acute lower respiratory tract infections and urinary tract infections are associat- 


\begin{tabular}{|c|c|c|c|c|c|c|c|c|c|c|}
\hline \multirow[t]{2}{*}{$\begin{array}{l}\text { Outcome and } \\
\text { Risk Period }\end{array}$} & \multicolumn{2}{|c|}{$\begin{array}{l}\text { Influenza Vaccination } \\
\qquad(\mathrm{N}=4010)\end{array}$} & \multicolumn{2}{|c|}{$\begin{array}{l}\text { Tetanus Vaccination } \\
\text { ( } \mathrm{N}=1889)\end{array}$} & \multicolumn{2}{|c|}{$\begin{array}{l}\text { Pneumococcal } \\
\text { Vaccination } \\
(\mathrm{N}=1686)\end{array}$} & \multicolumn{2}{|c|}{$\begin{array}{l}\text { Systemic Respiratory } \\
\text { Tract Infection } \\
(\mathrm{N}=5259)\end{array}$} & \multicolumn{2}{|c|}{$\begin{array}{l}\text { Urinary Tract Infection } \\
\qquad(\mathrm{N}=\mathbf{2 4 0 8 )}\end{array}$} \\
\hline & $\begin{array}{l}\text { No. of } \\
\text { Cases }\end{array}$ & $\begin{array}{c}\text { IR } \\
(95 \% \mathrm{CI})\end{array}$ & $\begin{array}{l}\text { No. of } \\
\text { Cases }\end{array}$ & $\begin{array}{c}\text { IR } \\
(95 \% \mathrm{CI})\end{array}$ & $\begin{array}{l}\text { No. of } \\
\text { Cases }\end{array}$ & $\begin{array}{c}\text { IR } \\
(95 \% \mathrm{CI})\end{array}$ & $\begin{array}{l}\text { No. of } \\
\text { Cases }\end{array}$ & $\begin{array}{c}\text { IR } \\
(95 \% \mathrm{CI})\end{array}$ & $\begin{array}{l}\text { No. of } \\
\text { Cases }\end{array}$ & $\begin{array}{c}\text { IR } \\
(95 \% \mathrm{CI})\end{array}$ \\
\hline \multicolumn{11}{|c|}{ Myocardial infarction } \\
\hline $1-3$ days & 11 & $\begin{array}{c}0.34 \\
(0.19-0.61)\end{array}$ & 1 & $\begin{array}{c}0.42 \\
(0.06-2.87)\end{array}$ & 2 & $\begin{array}{c}0.70 \\
(0.18-2.81)\end{array}$ & 61 & $\begin{array}{c}3.14 \\
(2.43-4.05)\end{array}$ & 12 & $\begin{array}{c}1.38 \\
(0.78-2.43)\end{array}$ \\
\hline 4-7 days & 34 & $\begin{array}{c}0.77 \\
(0.55-1.09)\end{array}$ & 2 & $\begin{array}{c}0.63 \\
(0.16-2.51)\end{array}$ & 2 & $\begin{array}{c}0.53 \\
(0.13-2.10)\end{array}$ & 59 & $\begin{array}{c}2.26 \\
(1.74-2.93)\end{array}$ & 18 & $\begin{array}{c}1.55 \\
(0.97-2.47)\end{array}$ \\
\hline 8-14 days & 71 & $\begin{array}{c}0.93 \\
(0.73-1.18)\end{array}$ & 7 & $\begin{array}{c}1.24 \\
(0.59-2.62)\end{array}$ & 9 & $\begin{array}{c}1.34 \\
(0.69-2.60)\end{array}$ & 78 & $\begin{array}{c}1.71 \\
(1.36-2.14)\end{array}$ & 35 & $\begin{array}{c}1.72 \\
(1.23-2.42)\end{array}$ \\
\hline 15-28 days & 146 & $\begin{array}{c}0.97 \\
(0.82-1.16)\end{array}$ & 7 & $\begin{array}{c}0.61 \\
(0.29-1.28)\end{array}$ & 14 & $\begin{array}{c}1.05 \\
(0.62-1.79)\end{array}$ & 131 & $\begin{array}{c}1.45 \\
(1.21-1.73)\end{array}$ & 51 & $\begin{array}{c}1.25 \\
(0.94-1.66)\end{array}$ \\
\hline 29-91 days & 607 & $\begin{array}{c}0.97 \\
(0.88-1.06)\end{array}$ & 58 & $\begin{array}{c}1.04 \\
(0.79-1.36)\end{array}$ & 79 & $\begin{array}{c}1.42 \\
(1.12-1.79)\end{array}$ & 488 & $\begin{array}{c}1.38 \\
(1.24-1.52)\end{array}$ & 172 & $\begin{array}{c}1.04 \\
(0.88-1.23)\end{array}$ \\
\hline \multirow{3}{*}{$\begin{array}{l}\text { Baseline } \\
\text { period }\end{array}$} & 3131 & 1.00 & 1812 & 1.00 & 1578 & 1.00 & 4339 & 1.00 & 2097 & 1.00 \\
\hline & \multicolumn{2}{|c|}{$\begin{array}{l}\text { Influenza Vaccination } \\
\qquad(N=4139)\end{array}$} & \multicolumn{2}{|c|}{$\begin{array}{l}\text { Tetanus Vaccination } \\
\qquad(\mathrm{N}=1355)\end{array}$} & \multicolumn{2}{|c|}{$\begin{array}{l}\text { Pneumococcal } \\
\text { Vaccination } \\
(\mathrm{N}=1117)\end{array}$} & \multicolumn{2}{|c|}{$\begin{array}{l}\text { Systemic Respiratory } \\
\text { Tract Infection } \\
(\mathrm{N}=6016)\end{array}$} & \multicolumn{2}{|c|}{$\begin{array}{l}\text { Urinary Tract Infection } \\
\qquad(\mathrm{N}=\mathbf{4 2 7 3})\end{array}$} \\
\hline & $\begin{array}{l}\text { No. of } \\
\text { Cases }\end{array}$ & $\begin{array}{c}\mathrm{IR} \\
(95 \% \mathrm{Cl})\end{array}$ & $\begin{array}{l}\text { No. of } \\
\text { Cases }\end{array}$ & $\begin{array}{c}\mathrm{IR} \\
(95 \% \mathrm{Cl})\end{array}$ & $\begin{array}{l}\text { No. of } \\
\text { Cases }\end{array}$ & $\begin{array}{c}\mathrm{IR} \\
(95 \% \mathrm{Cl})\end{array}$ & $\begin{array}{l}\text { No. of } \\
\text { Cases }\end{array}$ & $\begin{array}{c}\text { IR } \\
(95 \% \mathrm{Cl})\end{array}$ & $\begin{array}{l}\text { No. of } \\
\text { Cases }\end{array}$ & $\begin{array}{c}\text { IR } \\
(95 \% \mathrm{Cl})\end{array}$ \\
\hline \multicolumn{11}{|l|}{ Stroke } \\
\hline $1-3$ days & 19 & $\begin{array}{c}0.56 \\
(0.35-0.89)\end{array}$ & 3 & $\begin{array}{c}2.05 \\
(0.66-6.41)\end{array}$ & 2 & $\begin{array}{c}1.01 \\
(0.25-4.04)\end{array}$ & 70 & $\begin{array}{c}2.57 \\
(2.03-3.27)\end{array}$ & 37 & $\begin{array}{c}1.65 \\
(1.19-2.28)\end{array}$ \\
\hline 4-7 days & 33 & $\begin{array}{c}0.74 \\
(0.52-1.05)\end{array}$ & 1 & $\begin{array}{c}0.49 \\
(0.07-3.52)\end{array}$ & 3 & $\begin{array}{c}1.13 \\
(0.36-3.52)\end{array}$ & 80 & $\begin{array}{c}2.23 \\
(1.78-2.80)\end{array}$ & 52 & $\begin{array}{c}1.72 \\
(1.31-2.28)\end{array}$ \\
\hline 8-14 days & 56 & $\begin{array}{c}0.72 \\
(0.55-0.94)\end{array}$ & 2 & $\begin{array}{c}0.54 \\
(0.13-2.20)\end{array}$ & 3 & $\begin{array}{c}0.64 \\
(0.21-2.00)\end{array}$ & 94 & $\begin{array}{c}1.51 \\
(1.23-1.86)\end{array}$ & 72 & $\begin{array}{c}1.35 \\
(1.06-1.72)\end{array}$ \\
\hline 15-28 days & 105 & $\begin{array}{c}0.69 \\
(0.57-0.85)\end{array}$ & 5 & $\begin{array}{c}0.63 \\
(0.26-1.55)\end{array}$ & 10 & $\begin{array}{c}1.06 \\
(0.57-2.00)\end{array}$ & 145 & $\begin{array}{c}1.27 \\
(1.07-1.50)\end{array}$ & 124 & $\begin{array}{c}1.15 \\
(0.96-1.39)\end{array}$ \\
\hline 29-9l days & 516 & $\begin{array}{c}0.79 \\
(0.71-0.87)\end{array}$ & 38 & $\begin{array}{c}0.96 \\
(0.67-1.37)\end{array}$ & 46 & $\begin{array}{c}0.99 \\
(0.72-1.35)\end{array}$ & 501 & $\begin{array}{c}1.27 \\
(1.15-1.41)\end{array}$ & 470 & $\begin{array}{c}1.16 \\
(1.04-1.29)\end{array}$ \\
\hline $\begin{array}{l}\text { Baseline } \\
\text { period }\end{array}$ & 3396 & 1.00 & 1301 & 1.00 & 1053 & 1.00 & 4617 & 1.00 & 3472 & 1.00 \\
\hline
\end{tabular}

* The numbers of participants exposed to each type of vaccination or infection are shown in parentheses for each exposure. These include a small number who had a recorded myocardial infarction or stroke on the day of exposure that was not included in the analysis, because the events may have been recorded retrospectively. Incidence during the baseline period served as the reference category. IR denotes age-adjusted incidence ratio, and $\mathrm{Cl}$ confidence interval.

ed with a transient increase in the risk of a vascular event. The effect is seen for a first or a subsequent myocardial infarction or stroke and is most marked in the few days after infection. These findings, based on a very large set of data, support the link between acute infection and the risk of a vascular event, identify the magnitude of the association and its resolution over time, and offer insight into the factors that may determine the timing of acute vascular events.
No increase in the event rate was detected after influenza, tetanus, and pneumococcal vaccines.

In a study of the risk of vascular events after inflammatory stimuli, the potential for confounding is great, because participants who are vaccinated or in whom infections develop may differ from those who are not vaccinated or do not have infections. The advantage of the case-series method is that the influence of factors that vary among the participants, 
such as the baseline cardiovascular risk, is removed, because within-person comparisons are performed. The null hypothesis was that the event rates stay relatively constant with time and are not influenced by discrete external stimuli such as acute infections. Our finding of a substantial but short-lived increase in the incidence of vascular events after acute infection shows that the risk of a cardiovascular event fluctuates, and it is highly suggestive of a causal role for acute infections in triggering cardiovascular events.

There has been one previous case-control study of an association between acute respiratory tract infection and myocardial infarction that was based on the GPRD. ${ }^{24}$ The study included 475 persons who had a respiratory tract infection within the 12 months preceding a myocardial infarction and found that the risk of respiratory infection was increased by approximately a factor of three during the 10 days before the myocardial infarction but found no significant association with urinary tract infection. We confirmed this finding in a much larger group (more than 20,000 participants exposed to respiratory tract infections), using a different study design. In our study, the effect was similar for stroke, for both first and subsequent events, and, unlike the earlier smaller study, our study showed an increased risk after urinary tract infection. This finding is important, because it suggests that the effect of infections on cardiovascular risk may be generic and is not linked to specific types of infection. Furthermore, although certain cardiac presentations might be misdiagnosed as respiratory tract infection, this seems highly unlikely to be the case for urinary tract infection.

Our study was based on routine clinical data, and a potential weakness may be related to the quality of the data. However, the diagnosis of myocardial infarction in the GPRD has been examined in a subgroup of patients on the basis of electrocardiographic findings, elevated levels of cardiac enzymes, features of the history, or the receipt of fibrinolytic therapy, and the diagnosis was confirmed in more than 90 percent of the recorded cases. ${ }^{25,26} \mathrm{~A}$ diagnosis of stroke in the GPRD was checked by a review of hospital records and confirmed in 89 percent (78 of 88) of cases, and the incidence rates for stroke are similar to estimates obtained from other sources. ${ }^{19}$ The vaccination data recorded in the GPRD are likely to be of high quality, because there is close agreement between the prescribing data in this database and national data from the Prescrip- tion Pricing Authority; moreover, general practitioners have a financial incentive to record vaccines given. ${ }^{27}$

The incidence rates of myocardial infarction or stroke did not return fully to the baseline level within the three-month risk period after infection, a result that might be due to a greater likelihood that a diagnosis was recorded, but the magnitude of the residual increase was small (incidence ratio, 1.2 to 1.4) and even after taking this increase into account, the greatly increased rates seen in the period soon after infection remained significantly elevated. The effects of infection were not explained by seasonal patterns of exposure and vascular events.

One limitation of the study is that we did not know precisely the date of onset of infections but, rather, used the date of diagnosis. However, the majority of patients, even those with upper respiratory tract infections, visit their general practitioner within three days after the onset of symptoms, ${ }^{28}$ so that we are unlikely to have underestimated the duration of the increase in the risk of vascular events by more than a few days. An advantage of looking at vaccination as a stimulus is that we knew the date of exposure, but we saw no effect of vaccination on risk, probably because both the magnitude and the duration of the inflammation induced by vaccination are small, ${ }^{15}$ as compared with naturally occurring infection. ${ }^{29}$ The small protective effect seen after vaccination may have been due to the administration of vaccination when people were in periods of relatively good health.

The finding that two very different infectious processes in different organ systems are associated with a large but transient increase in the risk of cardiovascular events lends strong support to the concept that systemic inflammation itself alters the probability of the occurrence of a vascular event. The alternative explanation that there is some common acute precipitant of infection and vascular events seems less likely. We do not know whether the transient increase in risk is due to a short-term alteration of endothelial function or to other mechanisms, such as changes in plaque composition, white-cell activation, dehydration, or bed rest. Clearly, however, it will now be important to establish the mechanisms of and implications for prevention.

Our observations offer insight into the factors that may determine the timing of the onset of a vascular event in persons who have had a fairly stable degree of atherosclerosis for many years. The mild transient inflammation and associated suppression 
of endothelium-dependent relaxation induced by vaccination ${ }^{15}$ does not appear to translate into a detectable increase in the risk of vascular events.
Supported by a grant from the British Heart Foundation; a Medical Research Council Clinician Scientist Fellowship (to Dr. Smeeth); and a Wellcome Trust Advanced Fellowship (to Dr. Hubbard).

We are indebted to Chris Smith and Helena Viljoen for help with data processing.
REFERENCES

1. Alber DG, Powell KL, Vallance P, Goodwin DA, Grahame-Clarke C. Herpesvirus infection accelerates atherosclerosis in the apolipoprotein E-deficient mouse. Circulation 2000;102:779-85.

2. Lindahl B, Toss H, Siegbahn A, Venge $P$, Wallentin L. Markers of myocardial damage and inflammation in relation to long-term mortality in unstable coronary artery disease. N Engl J Med 2000;343:1139-47.

3. Danesh J, Wheeler JG, Hirschfield GM et al. C-reactive protein and other circulating markers of inflammation in the prediction of coronary heart disease. $\mathrm{N}$ Engl J Med 2004;350:1387-97.

4. Keaney JF Jr, Vita JA. The value of inflammation for predicting unstable angina. N Engl J Med 2002;347:55-7.

5. Brennan M-L, Penn MS, Van Lente F, et al. Prognostic value of myeloperoxidase in patients with chest pain. N Engl J Med 2003 349:1595-604.

6. Grau AJ, Boddy AW, Dukovic DA, et al Leukocyte count as an independent predic tor of recurrent ischemic events. Stroke 2004 35:1147-52.

7. Spodick DH, Flessas AP, Johnson MM Association of acute respiratory symptom with onset of acute myocardial infarction prospective investigation of 150 consecutive patients and matched control patients. Am J Cardiol 1984;53:481-2.

8. Saikku P, Leinonen M, Mattila K, et al Serological evidence of an association of a novel Chlamydia, TWAR, with chronic coronary heart disease and acute myocardial infarction. Lancet 1988;2:983-6.

9. Mattila KJ, Nieminen MS, Valtonen VV et al. Association between dental health and acute myocardial infarction. BMJ 1989;298 779-81.

10. Mattila KJ. Viral and bacterial infections in patients with acute myocardial infarction. J Intern Med 1989;225:293-6.
11. Syrjanen J, Valtonen VV, Iivanainen M, Kaste M, Huttunen JK. Preceding infection as an important risk factor for ischaemic brain infarction in young and middle aged patients. Br Med J (Clin Res Ed) 1988;296:1156-60.

12. Valtonen V, Kuikka A, Syrjanen J. Thrombo-embolic complications in bacteraemic infections. Eur Heart J 1993;14:Supp $\mathrm{K}: 20-3$.

13. Kinlay S, Ganz P. Role of endothelia dysfunction in coronary artery disease and implications for therapy. Am J Cardiol 1997 80:11-I-16-I

14. Chan NN, Colhoun HM, Vallance P. Cardiovascular risk factors as determinants of endothelium-dependent and endotheliumindependent vascular reactivity in the general population. J Am Coll Cardiol 2001;38: 1814-20.

15. Hingorani AD, Cross J, Kharbanda RK, et al. Acute systemic inflammation impair endothelium-dependent dilatation in humans. Circulation 2000;102:994-9.

16. Farrington CP, Nash J, Miller E. Case series analysis of adverse reactions to vaccines: a comparative evaluation. Am J Epidemio 1996;143:1165-73. [Erratum, Am J Epidemiol 1998;147:93.]

17. Walley T, Mantgani A. The UK Genera Practice Research Database. Lancet 1997; 350:1097-9.

18. Key health statistics from general prac tice 1996. Series MB6. No. 1. London: Office for National Statistics, 1998.

19. Gibbs RG, Newson R, Lawrenson R, Greenhalgh RM, Davies AH. Diagnosis and initial management of stroke and transien ischemic attack across UK health regions from 1992 to 1996: experience of a national primary care database. Stroke 2001;32 1085-90.

20. Farrington CP. Relative incidence estimation from case series for vaccine safety evaluation. Biometrics 1995;51:228-35.
21. Idem. Control without separate controls: evaluation of vaccine safety using case-only methods. Vaccine 2004;22:2064-70.

22. Tata LJ, WestJ, Harrison T, Farrington $P$, Smith C, Hubbard R. Does influenza vaccination increase consultations, corticosteroid prescriptions, or exacerbations in subjects with asthma or chronic obstructive pulmonary disease? Thorax 2003;58:835-9.

23. Woodhouse PR, Khaw KT, Plummer M, Foley A, Meade TW. Seasonal variations of plasma fibrinogen and factor VII activity in the elderly: winter infections and death from cardiovascular disease. Lancet 1994;343: 435-9.

24. Meier CR, Jick SS, Derby LE, Vasilakis C, Jick H. Acute respiratory-tract infections and risk of first-time acute myocardial infarction. Lancet 1998;351:1467-71.

25. Jick H, Derby LE, Gurewich V, Vasilakis C. The risk of myocardial infarction associated with antihypertensive drug treatment in persons with uncomplicated essential hypertension. Pharmacotherapy 1996;16: 321-6.

26. Jick H, Vasilakis C, Derby LE. Antihypertensive drugs and fatal myocardial infarction in persons with uncomplicated hypertension. Epidemiology 1997;8:446-8.

27. Hollowell J. The General Practice Research Database: quality of morbidity data. Popul Trends 1997;87:36-40.

28. Little P, Williamson I, Warner G, Gould C, Gantley M, Kinmonth AL. Open randomised trial of prescribing strategies in managing sore throat. BMJ 1997;314:722-7.

29. Melbye H, Hvidsten D, Holm A, Nordbo SA, Brox J. The course of C-reactive protein response in untreated upper respiratory tract infection. Br J Gen Pract 2004;54: 653-8.

Copyright @ 2004 Massachusetts Medical Society. 